\title{
Tissue Factor is an Angiogenic Mediator that Induces both Vascular Endothelial Growth Factor (VEGF) and Basic Fibroblast Growth Factor (bFGF)
}

\author{
Jung A Lee, †'Jae Eun Park, Sewook Hwang, Do Hee Lee, Sung Goo Park, \\ Byoung Chul Park, ${ }^{\star}$ and Sayeon Cho ${ }^{\dagger, *}$ \\ "College of Pharmacy, Chung-Ang University, Seonl 156-756, Korea. "E-mail: sycho@can.ac.kr \\ Proteome Research Laboratory, Korea Research Institute of Bioscience and Biotechnology, Daejeon 305-333, Korea \\ "E-mail: parkbc@kribb.re.kr \\ Received May 30, 2006
}

\begin{abstract}
Key Words : Angiogenesis, Tissue factor, Vascular endothelial growth factor (VEGF), Basic fibroblast growth
\end{abstract} factor (bFGF)

Acute myeloid leukemia (AML) is a biologically heterogeneous disease of the hematopoietic system characterized by a block in differentiation of immature blast cells in bone marrow. Recent studies suggest that angiogenesis in bone marrow plays a critical role in leukemogenesis and thus antianglogenic strategies are considered for the treatment of AML. ${ }^{1}$ Angiogenesis, the process of new blood vessels from pre-existing vessels, plays an important role in various physiological and pathological conditions. Angiogenesis in physiological processes is involved in embryonic development, growth, and wound healing. The process is a multi-step mechanism that encompasses activation, migration, and proliferation of endothelial cells. Angiogenesis can occur when the balance is changed towards a decreased level of inhibitors of angiogenesis or an increased level of angiogenic activators such as vascular endothelial growth factor (VEGF) or basic fibroblast growth factor (bFGF). ${ }^{2}$ Tumors need angiogenesis for growth that requires the supply of oxygen and other essential nutrients as well as for metastasis. Since the process is involved in cancer cell growth and metastasis, it has been the central focus on cancer treatment and prevention. More than 20 angiogenic factors and 300 anti-angiogenic factors have been identified so far. Of them, VEGF and bFGF have a prominent activity in angiogenesis. ${ }^{3}$ Transcriptional activation of VEGF gene is mediated by a variety of cellular transcription factors including hypoxia-inducible factor-1 (HIF-1) and other transcription factors such as NF- $\mathrm{BB}$ and SP1. ${ }^{4-7}$ Especially, HIF-1 plays an important role in VEGF induction. Since angiogenesis is controlled by multiple regulation mechanisms, more endogenous anigogenic regulators might be involved in the process.

In the present study, we studied the effect of tissue factor (TF) on angiogenesis to elucidate the regulation mechanism of angiogenesis induced by TF. TF is the essential receptor for factor VII/VIIa and is the primary initiator of blood coagulation in vivo. However, recent studies have suggested that TF regulates the angiogenesis in tumors. ${ }^{8}$ Also, the expression of $\mathrm{TF}$ in the hepatocarcinoma ( $\mathrm{HCC}$ ) tissue sample correlated significantly with VEGF level and tumor microvessel density. ${ }^{9}$ However, the functional connection between $\mathrm{TF}$ and the angiogenic process is still unclear.

Since endothelial cell migration through extracellular matrix is one of the essential steps for neovascularization, we first investigated the effect of TF on endothelial cell migration. For these experiments, we transfected HEK 293 cells with a plasmid bearing TF gene and Zeocin marker for selection. After selection of cells stably expressing TF in Zeocin-containing medium and confirmation of protein expression, Conditioned medium (CM) was collected from Zeocin-resistant cells for HUVEC migration assays. In a chemotaxis chamber, HUVECs were treated with CM from stably transfected cells for $2 \mathrm{~h}$ and HUVECs that had been migrated through membrane pores were counted under microscope. We used VEGF $(20 \mathrm{ng} / \mathrm{mL})$ as a positive control. CM from TF-overexpressing HEK 293 cells (TFCM) showed significantly induced migratory effect, compared to that of control-CM (CM obtained from empty plasmid-transfected cells) (Fig. 1A). This result suggests that CM from cells stably overexpressing TF (TF-CM) has the biological activity that can induce endothelial cell migration.

In vitro angiogenesis was assessed by endothelial cell tube fomation as well as endothelial cell migration. Tube fomation is a major process of angiogenesis. The endothelial cells begin to divide, and they migrate out through the dissolved holes of the existing vessel towards the diseased tissue. As the vessel extends, the tissue is remolded around the vessel. Then sprouting endothelial cells roll up to form a blood vessel tube. The tube formation assay is designed to analyze an endothelial cell's ability to develop a blood vessel. To determine whether TF functions for the HUVEC tube formation, we examined the ability of TF to promote the formation of capillary-like structures of HUVECs on Matrigel. We used bFGF $(10 \mathrm{ng} / \mathrm{mL})$ as a positive control. CM collected from TF-overexpressing HEK 293 cells (TF$\mathrm{CM}$ ) caused an increase in capillary-like structures, compared to control-CM (Fig. 1B). Therefore, these data indicate that TF has ability of inducing tube formation of endothelial cells.

We then measured the protein levels of VEGF and bFGF in CM to investigate whether their production is involved in TF-stimulated HUVEC tube formation since they are ubiquitous and potent activators of angiogenesis. Using 

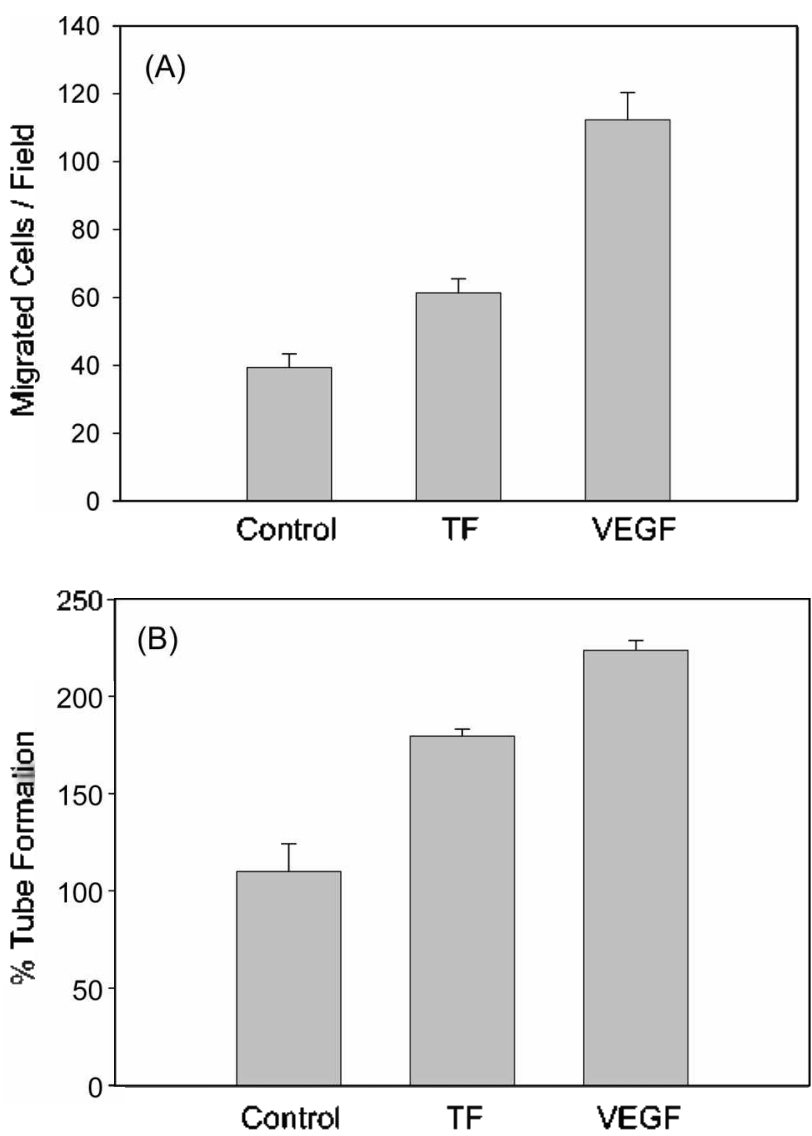

Figure 1. HUVEC migration and tube fonnation assays with TF$\mathrm{CM}(\Lambda) . \mathrm{TF}-\mathrm{CM}$ stimulates endothelial cell migration. HUVECs were incubated with control-CM or TF-CM for $2 \mathrm{~h}$. After fixation and staining, the number of migrated cells was determined by counting two regions of each well under a microscope. VEGF (20 $n g / n L$ ) was used as a positive control. (B). HUVECs were collected and seeded on Matrigel-coated plates at a density of $2.4 \times 10^{4}$ cells/well and then incubated with control-CM or TFCM. $\Lambda$ s a positive control, bFGF $(10 \mathrm{ng} / \mathrm{mL})$ was used. $\Lambda$ fter $18 \mathrm{~h}$, ficlds from each sanple were photographed. Total tube areas were analyzed and quantitated by the Scion Image program. The control tube areas were defined as $100 \%$ tube formation, and the percent increase in tube formation as compared with control was calculated for cach sample.

ELISA kit, we found that TF-overexpressing cells released significantly more VEGF and bFGF than control cells (Fig. $2 \mathrm{~A}$ and $\mathrm{B}$ ), suggesting that the increased levels of secreted VEGF and bFGF are associated with TF-induced angiogenic activity involved in migration and tube formation.

Since TF-CM contains increased level of VEGF that is a target of HIF-1, we carried out HRE-luciferase reporter assay to examine whether TF regulates the transactivation activity of HIF-1 that is stabilized at hypoxia and plays a key role in VEGF transcription. TF-overexpressing cells were transfected with pHRE-Luc reporter plasmid and either grown under normoxic condition or treated with a hypoxiamimicking reagent ( $125 \mu \mathrm{M} \mathrm{CoCl}$ ). As shown in Figure 3, TF-overexpressing cells resulted in about 4-fold increase of the luciferase activity under normoxic condition and about 12-fold increase under hypoxia-mimicking condition, compared
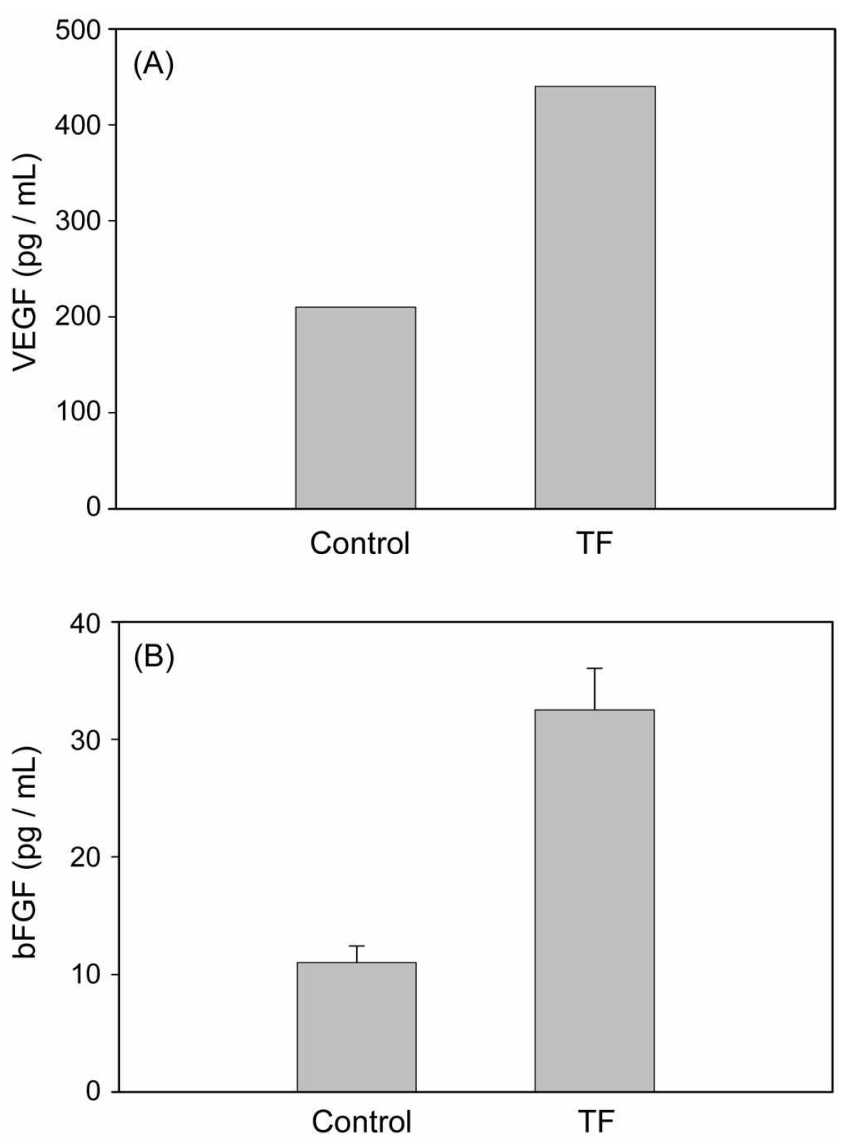

Figure 2. TF increases the levels of VEGF and bFGF in CM. $(\Lambda)$ Control or TF-overexpressing stable cells were incubated in serumfree M199 medium for $20 \mathrm{~h}$, and aliquots of the CM were collected. The level of VEGF in CM was measured by ELIS $A$ as described in Material and Methods. (B) The level of bFGF in CM was measured by ELISA.

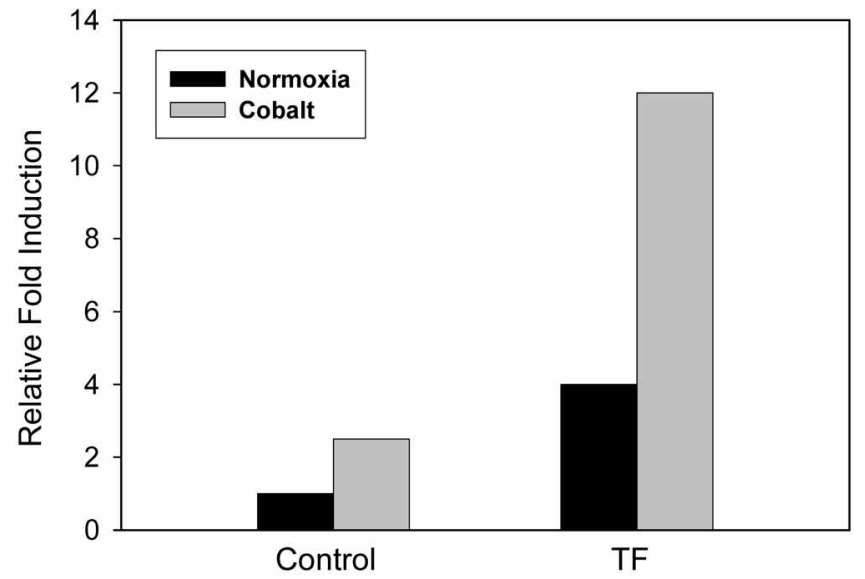

Figure 3. TF enhances the transactivation activity of HIF-1. HEK 293 cells that constitutively express TF and control cells that contain enpty peDNA3.1/Zco plasinid were transfected by lipofectarnine with $1 \mu \mathrm{e}$ each of the reporter plasmid pHRE-Luc and 0.5 $\mu \mathrm{g}$ of pCMV $/ \beta$ gal. Cells were treated with $125 \mu \mathrm{M} \mathrm{CoCl} \mathrm{L}_{2}$ for $24 \mathrm{~h}$. Luciferase activities were normalized to $\beta$-galactosidase activities.

to control cells grown at normoxia. Interestingly, the relative ratios of luciferase activities from TF-overexpressing cells 
incubated under hypoxia-mimicking and normoxic conditions are similar to those of control cells, respectively, suggesting that $\mathrm{TF}$ activates $\mathrm{HIF}-1$ activity at both normoxia and hypoxia. Taken together, these results suggest that TF activates the HIF-1 transactivation activity and that its activatory function is oxygen-independent.

In conclusion, the results of this study suggest that TF mediates angiogenesis by inducing both VEGF and bFGF. The induction of VEGF by TF may involve HIF-1-mediating pathway, at least in part. Since TF, however, induces the transactivation activity of HIF-1 irrespective of oxygen level and thus leads to the increase of VEGF secretion at normoxia, these results indicate that TF may be involved in oxygen-independent HIF-1 regulation pathway in some degree. Since mitogen-activated protein kinase (MAPK) pathways have been known to be activated by $\mathrm{TF}^{10}$ and activates HIF-1, it is conceivable that TF activates MAPK pathways and they, in tum, activates HIF-1 for VEGF production. This observation provides evidence that TF might be a candidate for developing anti-cancer agent in tumor growth and angiogenesis.

\section{Experimental Section}

Cell culture. Human embryonic kidney (HEK) 293 and human umbilical vein endothelial cells (HUVECs) were routinely maintained as previously described."

Plasmid constructs. The C-terminal hemagglutinin (HA)tagged gene for expression in mammalian cells was constructed by polymerase chain reaction, followed by cloning into the pcDNA3.1/Zeo-HA plasmid. The luciferase reporter plasmid ( $\mathrm{pHRE}-\mathrm{Luc}$ ) containing the promoter of HIF- $1 \alpha$ was purchased from Stratagene.

Transfection and selection of TF-overexpressing cells. HEK 293 cells were transfected by lipofectamine reagent (Invitrogen) with $1 \mu \mathrm{g}$ each of pcDNA3.1/Zeo-HA-TF or pcDNA3.1 Zeo-empty vector as a control. After $48 \mathrm{~h}$, transfected cells were selected for two weeks in DMEM containing $10 \%$ FBS and Zeocin $(200 \mu \mathrm{g} / \mathrm{mL})$. After 15 days of selection, resistant cells arising in dishes were propagated in low level $(150 \mu \mathrm{g} / \mathrm{mL})$ of Zeocin. The overexpressed TF in stably transfected cells was confirmed by immunoblotting.

Preparation of conditioned medium (CM). Confluent cells were washed and grown in serum free M199 medium. After $20 \mathrm{~h}$ of incubation, CM was collected and centrifuged at $500 \times \mathrm{g}$ for $10 \mathrm{~min}$ and then at $800 \times \mathrm{g}$ for $20 \mathrm{~min}$ to remove debris. The resultant $\mathrm{CM}$ was immediately used for migration and tube fomation assays or stored at $-70^{\circ} \mathrm{C}$ until use.

HUVEC migration and tube formation assays. Endothelial cell migration and tube formation assays were carried out as previously described. ${ }^{12}$

Luciferase assays. TF-overexpressing HEK 293 cells and control HEK 293 cells were grown to $50-80 \%$ confluence in $60 \mathrm{~mm}$ cell culture dish, transfected by lipofectamine with 1 $\mu \mathrm{g} \mathrm{pHRE}-\mathrm{Luc}$ and $\mathrm{pCMV} / \beta$-gal, and assayed as previously described. ${ }^{13}$

Determination of the VEGF and bFGF levels. The amounts of VEGF or bFGF proteins in the CM from the cells were determined using an enzyme-linked immunosorbent assay kit (R\&D Systems, Minneapolis, MN) according to the manufacturer's instructions. Quantification of VEGF or bFGF expression level was analyzed in equal densities of cells.

Acknowledgements. This work was supported by a grant of the Korea Health 21 R\&D Project, Ministry of Health \& Welfare, Republic of Korea (01-PJ10-PG6-01GN16-(0005).

\section{References}

I. Hatfield, K. J.; Olsnes, A. M.; Gjertsen, B. T.; Brusend, O. Cw Cancer Drig Targets $\mathbf{2 0 0 5}, 5,229$.

2. Folkman, J. Nat. Med. 1995, 1, 27.

3. Yoshiji, H.; Kuriyama, S.; Yoshii, J.; Ikenaka, Y.; Noguchi, R.; Hicklin. D. J.; Huber, J.; Nakatani, T.; Tsujinoue, H.; Yanase, K.; Imazu, H.; Fukui, H. Hepatology 2002, 35, 834.

4. Ravi, R.; Mookerjee, B.; Bhujwalla, Z. M.; Sulter, C. H.; Arlemov, D.; Zeng, Q.; Dillehay, L. E.; Madan, A.; Semenza, G. L.; Bedi, A. Genes, Dev: 2000, 14, 34.

5. Fukuda, R.; Kelly, B.; Semenza, G. L. Cancer Res. 2003, 63, 2330.

6. Shukla, S.; MacLennan, G T.; Fu, P.; Patel, J.; Marengo, S. R.; Resnick, M. I.; Gupla, S. Neoplasia 2004, 6, 390.

7. Loureiro, R. M.; D'Amore, P. A. Cytokine Growth Factor Rev: $2005,16,77$.

8. Zhang. Y.; Deng. Y.; Luther, T.; Muller, M.; Ziegler, R.; Waldhen, R.; Stern, D. M.; Nawroth, P. P. J. Clin. Invest. 1994, 94, 1320.

9. Poon, R. T.; Lau, C. P.; Ho, J. W.; Yu, W. C.; Fan, S. T.; Wong. J. Clin. Cancer Res, $2003,9,5339$.

10. Versleeg, H. H.; Spek, C. A.; Peppelenbosch, M. P.; Richel, D. J. Mol, Med, 2004, 10,6.

11. Cho, S. Bull. Korean Chem. Soc. 2005, 26, 1823.

12. Kim, C. W.; Lee, H. M.; Lee, T. H.; Kang, C.; Kleinman, H. K.; Gho, Y. S. Cancer Res. 2002, 62,6312.

13. Park, J. E.; Lee do, H.; Lee, J. A.; Park, S. G.; Kim, N. S.; Park, B. C.; Cho, S. Biochem. Biophys, Res. Commm 2005, 337 , 1283. 